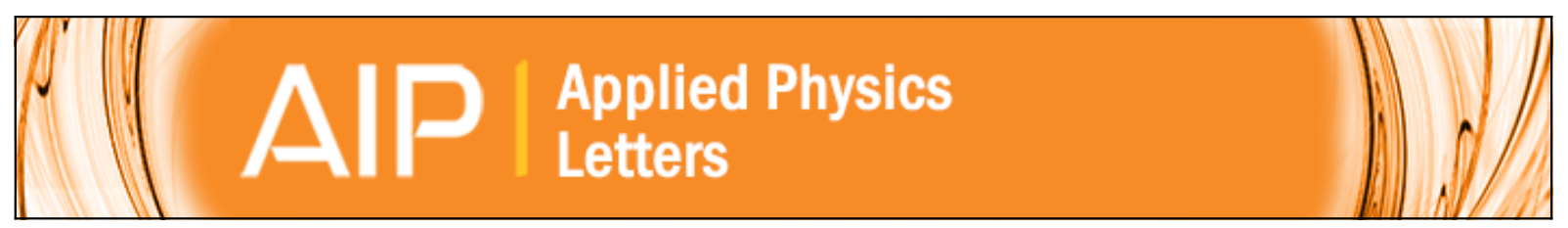

\title{
High quality factor indium oxide mechanical microresonators
}

Javier Bartolomé, Ana Cremades, and Javier Piqueras

Citation: Applied Physics Letters 107, 191910 (2015); doi: 10.1063/1.4935708

View online: http://dx.doi.org/10.1063/1.4935708

View Table of Contents: http://scitation.aip.org/content/aip/journal/apl/107/19?ver=pdfcov

Published by the AIP Publishing

\section{Articles you may be interested in}

Suspended plate microresonators with high quality factor for the operation in liquids

Appl. Phys. Lett. 104, 191906 (2014); 10.1063/1.4875910

High quality factor nanocrystalline diamond micromechanical resonators limited by thermoelastic damping

Appl. Phys. Lett. 104, 151903 (2014); 10.1063/1.4871803

Microcrystalline diamond micromechanical resonators with quality factor limited by thermoelastic damping Appl. Phys. Lett. 102, 071901 (2013); 10.1063/1.4793234

High Q factor for mechanical resonances of batch-fabricated SiC nanowires

Appl. Phys. Lett. 90, 043113 (2007); 10.1063/1.2432257

Mechanical dissipation in tetrahedral amorphous carbon

J. Appl. Phys. 97, 023517 (2005); 10.1063/1.1821638

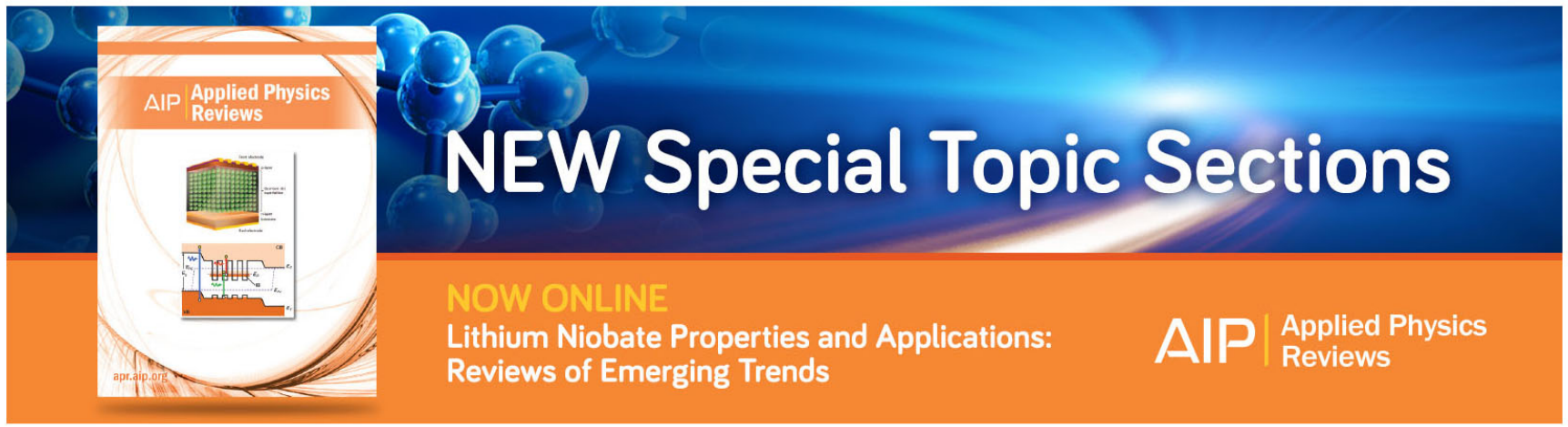




\title{
High quality factor indium oxide mechanical microresonators
}

\author{
Javier Bartolomé, ${ }^{\text {a),b) }}$ Ana Cremades, and Javier Piqueras \\ Department of Materials Physics, Faculty of Physics, Universidad Complutense de Madrid, \\ 28040 Madrid, Spain
}

(Received 22 September 2015; accepted 2 November 2015; published online 13 November 2015)

\begin{abstract}
The mechanical resonance behavior of as-grown $\mathrm{In}_{2} \mathrm{O}_{3}$ microrods has been studied in this work by in-situ scanning electron microscopy (SEM) electrically induced mechanical oscillations. Indium oxide microrods grown by a vapor-solid method are naturally clamped to an aluminum oxide ceramic substrate, showing a high quality factor due to reduced energy losses during mechanical vibrations. Quality factors of more than $10^{5}$ and minimum detectable forces of the order of $10^{-16}$ $\mathrm{N} / \mathrm{Hz}^{1 / 2}$ demonstrate their potential as mechanical microresonators for real applications. Measurements at low-vacuum using the SEM environmental operation mode were performed to study the effect of extrinsic damping on the resonators behavior. The damping coefficient has been determined as a function of pressure. (C) 2015 AIP Publishing LLC.
\end{abstract}

[http://dx.doi.org/10.1063/1.4935708]

During the last decade, the study of mechanical micro and nanoresonators has attracted a great interest due to their high potential in the field of ultrasensitive chemical, ${ }^{1}$ biolog$\mathrm{ical}^{2}{ }^{2}$ and force ${ }^{3,4}$ sensing, with sensitivities reaching the single molecule detection. ${ }^{5}$ They also found applications as micro and nanoelectromechanical systems (MEMS and NEMS) in devices such as nanoswitches, ${ }^{6}$ nanoradios, ${ }^{7}$ or mechanical energy harvesters. ${ }^{8}$ Besides, in recent years a renewed interest has appeared in fundamental studies of the light-matter interaction through the optomechanical coupling, ${ }^{9,10}$ which could lead to optically enhanced quality factor mechanical resonators. ${ }^{11,12}$ Depending on the desired application, different materials and configurations are used to fabricate the resonators. High quality factor cantileverlike resonators are typically made of diamond ${ }^{13-15}$ or silicon, ${ }^{16,17}$ either single-crystal or polycrystalline, although many other materials, such as silicon carbide, ${ }^{3}$ silicon nitride, ${ }^{16}$ carbon nanotubes, ${ }^{4}$ or polymeric materials, ${ }^{18}$ have been also investigated. However, diamond cantilevers of sufficient quality are expensive and difficult to obtain, while $\mathrm{Si}$ lacks of optical transparency, of interest for the study of the optomechanical coupling. In comparison, indium oxide is a wide bandgap semiconductor characterized for its high transparency and refractive index in the visible range $(\mathrm{n} \sim 2)$ and n-type intrinsic conductivity. It can be grown over different substrates in the form of elongated micro and nanostructures such as rods, ${ }^{19}$ wires, ${ }^{20,21}$ and belts. ${ }^{22,23}$ These structures present waveguiding behavior and it has been recently demonstrated that indium oxide microrods are able to sustain optical resonant modes ${ }^{19}$ making this material an interesting alternative for the study of the optomechanical coupling or for the fabrication of transparent MEMS. ${ }^{24}$ Furthermore, its high thermal and chemical stability ${ }^{25}$ makes it a good choice for its use in harsh environments. Notwithstanding, reports

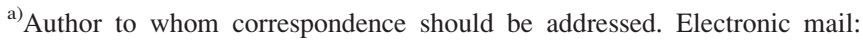
j.bartolome@fis.ucm.es

b) Present address: Paul-Drude-Institut für Festkörperelektronik, Hausvogteiplatz 5-7, 10117 Berlin, Germany.
}

on mechanical properties of indium oxide ${ }^{26-29}$ and on its suitability for mechanical microresonators are very scarce. ${ }^{30}$

In a previous work, we studied by an in-situ scanning electron microscopy (SEM) technique the electrically induced mechanical oscillations of indium oxide microrods fixed with silver paint to a $\mathrm{Si}$ substrate in a cantilever-like configuration. ${ }^{30}$ In that work, the feasibility of the in-situ SEM technique for the determination of the resonance frequency and Young's modulus of the structures was assessed and a quality factor (Q-factor o simply $Q$ ) for the studied rods of more than $10^{3}$ was obtained. However, it is well known that the clamping condition of mechanical resonators highly influences their Q-factor as it is a major source of energy losses. ${ }^{16,18}$ Microrods grown by evaporationdeposition methods are naturally clamped to the substrate, which should lead to reduced energy losses during mechanical vibrations as compared to those clamped with silver paint or any other adhesive. In order to study the effect of clamping on the $\mathrm{Q}$ factor, the resonance behavior of as-grown $\mathrm{In}_{2} \mathrm{O}_{3}$ microrods has been studied in this work by in-situ SEM electrically induced mechanical oscillations.

Indium oxide microrods were fabricated by a thermal evaporation-deposition method using $\mathrm{In}_{2} \mathrm{~S}_{3}$ powder as precursor by the vapor-solid method described in Ref. 19. After the thermal treatment, the surface of the alumina boat is covered by indium oxide rods (Figure 1(a) and inset in Figure 1(b)) with lateral dimensions ranging from several hundreds of nanometers to few tens of microns, and lengths from several hundreds of microns to more than one millimeter. Hereinafter, natural clamping refers to the clamping of the as-grown rods to the boat. The microrods were characterized by means of X-ray diffraction (XRD), performed with a PANalytical's X'Pert MPD diffractometer with $\mathrm{Cu} \mathrm{K}_{\alpha}$ radiation. Their morphology was studied by SEM with an FEI Inspect variable pressure SEM and their chemical composition was assessed by means of X-ray energy dispersive spectroscopy (EDS) using a Bruker AXS XFlash 4010 detector coupled to a Leica 440 Stereoscan SEM. The crystalline orientation of the rods was studied by means of electron 

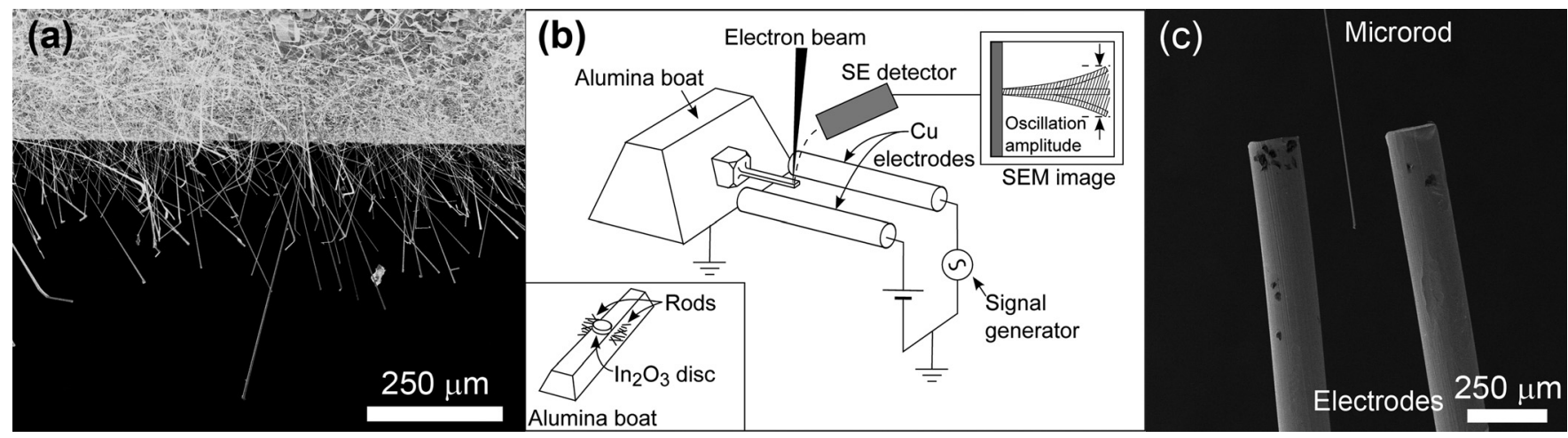

FIG. 1. (a) SEM image of the surface of the alumina boat, covered by $\mathrm{In}_{2} \mathrm{O}_{3}$ microrods. (b) Schematic of the setup employed for the in-situ SEM electrically induced mechanical resonance measurements. For the sake of clarity, only one rod is represented in the picture. Inset shows a schematic of the alumina boat with indium oxide rods on its surface. (c) SEM image of a microrod between the $\mathrm{Cu}$ electrodes.

backscattered diffraction (EBSD) with a Bruker e-FlashHR ${ }^{+}$ detector coupled to the FEI Inspect SEM.

To study the mechanical response of the rods with natural clamping, the alumina boat with the microrods was placed inside the SEM chamber, and vibration of the rods was induced in-situ by an alternating electric field ${ }^{31}$ applied between two cylindrical $\mathrm{Cu}$ electrodes as shown in the schematic of Figure 1(b). An SEM image of an indium oxide rod with natural clamping placed between both cylindrical electrodes is shown in Figure 1(c). Frequency $(v)$ and voltage drop $\left(V_{A C}\right)$ were controlled by a Standford Research System SR830 DSP Lock-In Amplifier, while the vibration amplitude of the rods was monitored by means of the SEM imaging system. The cylindrical electrodes configuration produces an inhomogeneous electric field, which may lead to the occurrence of parametric oscillations ${ }^{32}$ and/or changes in the measured resonance frequency. ${ }^{33}$ These problems can be avoided using plane-parallel electrodes as demonstrated in Ref. 30; however, the large size of the alumina boat strongly limits the electrical field applied between the electrodes, therefore hindering the measurements, and the cylindrical electrodes enhance the selectivity of the technique since only the rod under study is placed between the electrodes. Thus, in order to check the validity of the data measured with the cylindrical electrodes, we first compared the results obtained in several test rods glued with silver paste to a Si substrate in a cantilever-like shape using this configuration and the plane-parallel configuration assessed in Ref. 30. The results were found to deviate less than $0.5 \%$ between both configurations.

Figures 2(a) and 2(b) show, respectively, a representative image of the tip and the bottom part of different indium oxide microrods. All the studied rods present rectangular cross sections and lengths of several hundreds of microns. Their cross sectional dimensions range from hundreds of nanometers to several microns, and usually decrease from the tip to the bottom. The base of the rods usually consists of a thicker $\operatorname{In}_{2} \mathrm{O}_{3}$ crystallite of several tens of microns in size which rapidly tapers to give rise to the rods, forming a natural clamping with certain curvature radius (see inset in Figure 2(b)). Figures 2(c) and 2(d) show a representative EBSD pattern recorded on a lateral face of a rod and its corresponding pole figure along the [110] axis. From the pole figure, it can be concluded that the lateral faces correspond to $\{110\}$ crystalline planes, and therefore the growth of the structure takes place along the [100] direction. This differs from other reported rectangular indium oxide rods, wires, and belts grown along the [100] direction, which have been usually described as enclosed by $\{100\}$ faces. ${ }^{34,35}$ One of the main factors that determine the final facets of a crystal is the surface energy of its different crystallographic planes which depends on the environmental conditions, such as supersaturation, indium or oxygen rich conditions, temperature, etc., and therefore, the final faceting of the rods can change between $\{110\}$ and $\{100\}$ planes depending on the growth conditions.

For the study of mechanical resonances, three different rods, hereinafter called as microrods $\mathrm{A}, \mathrm{B}$, and $\mathrm{C}$, were selected, whose measured geometrical and physical parameters are summarized in Table I. The vibration of the rods as a

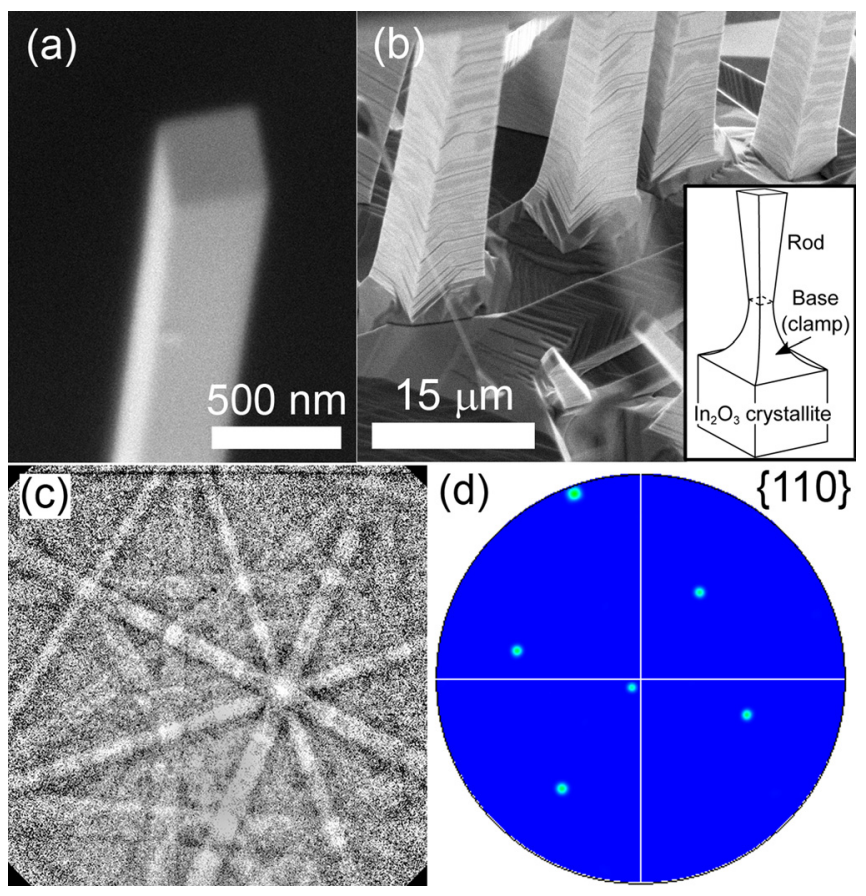

FIG. 2. (a) and (b) Representative SEM images of the tip and the bottom parts, respectively, of different indium oxide microrods. Inset: Schematic of the base (clamp) of the microrods. Tapering of the rod and its base has been magnified for the sake of clarity. (c) and (d) Representative EBSD pattern recorded from the surface of the rods and its corresponding pole figure along the $[110]$ axis, showing the $\{110\}$ planes. 
TABLE I. Physical and geometrical parameters of the studied rods: $l$ is the length, $S_{B}$ and $S_{T}$ are the cross sectional area at the bottom and the tip, respectively, $v$ is the resonance frequency, $\tau$ is the oscillator decay constant, $Q$ is the quality factor, $k_{r o d}$ is the spring constant, and $F_{\min }$ is the minimum detectable force.

\begin{tabular}{lccc}
\hline \hline & $A$ & $B^{\mathrm{a}}$ & $C^{\mathrm{a}}$ \\
\hline Cross sectional shape & Rectangular & Rectangular & Rectangular \\
Growth direction & {$[100]$} & {$[100]$} & {$[100]$} \\
$l(\mu \mathrm{m})$ & $494 \pm 9$ & $438 \pm 9$ & $357 \pm 9$ \\
$S_{B}\left(\mu \mathrm{m}^{2}\right)$ & $1.769 \pm 0.027$ & $1.369 \pm 0.023$ & $1.118 \pm 0.015$ \\
$S_{T}\left(\mu \mathrm{m}^{2}\right)$ & $15.210 \pm 0.078$ & $29.48 \pm 0.11$ & $5.639 \pm 0.035$ \\
$v_{0}(\mathrm{~Hz})$ & $1457.9 \pm 0.1$ & $3455 \pm 1$ & $5069 \pm 1$ \\
$\tau(\mathrm{s})^{\mathrm{b}}$ & $25.46 \pm 0.49$ & $45.61 \pm 0.47$ & $3.95 \pm 0.39$ \\
$Q^{\mathrm{b}}$ & $(1.166 \pm 0.022) \times 10^{5}$ & $(4.951 \pm 0.051) \times 10^{5}$ & $(6.29 \pm 0.62) \times 10^{4}$ \\
$k_{\text {rod }}(\mathrm{N} / \mathrm{m})$ & $4.54 \times 10^{-3}$ & $2.29 \times 10^{-2}$ & $8.78 \times 10^{-3}$ \\
$F_{\text {min }}\left(\mathrm{N} / \mathrm{Hz}^{1 / 2}\right)$ & $2.65 \times 10^{-16}$ & $1.88 \times 10^{-16}$ & $2.70 \times 10^{-16}$ \\
\hline \hline
\end{tabular}

${ }^{a}$ Values of $k_{\text {rod }}$ and $F_{\min }$ have been calculated using the effective mass obtained with Eq. (5).

${ }^{\mathrm{b}}$ Measured in high-vacuum conditions.

function of the frequency of the applied voltage was directly monitored in the SEM (Figure 3(a)). The frequency was tuned to find the resonance condition of the oscillating rod, and the oscillation amplitude was measured from the SEM images. The resonance frequency, corresponding to the maximum vibration amplitude, was, for the three studied rods, of the order of $\mathrm{kHz}$, as shown in Table I. Decay curves were obtained by driving the rods to the resonance condition and then turning the AC voltage off. Evolution of the rod's free oscillation amplitude was video-recorded and analyzed to obtain the experimental data. In order to ensure that the SEM electron beam was not interfering with the rods oscillations, different measurements were performed by turning the beam off before starting the decay experiment and turning it on again after a short period of time so it was still possible to record some part of the decay curves. All the obtained curves (normalized to the initial resonant amplitude) matched well with those obtained when leaving the electron beam on during the whole experiment. Figures 3(b)-3(d) show the decay curves obtained for each microrod, measured at room temperature and $10^{-6}$ Torr. The oscillator decay constant, $\tau$, is determined by fitting the experimental amplitude decay curves of the rods to an exponential function. ${ }^{16}$ The obtained $\tau$ values are displayed in their corresponding decay curves

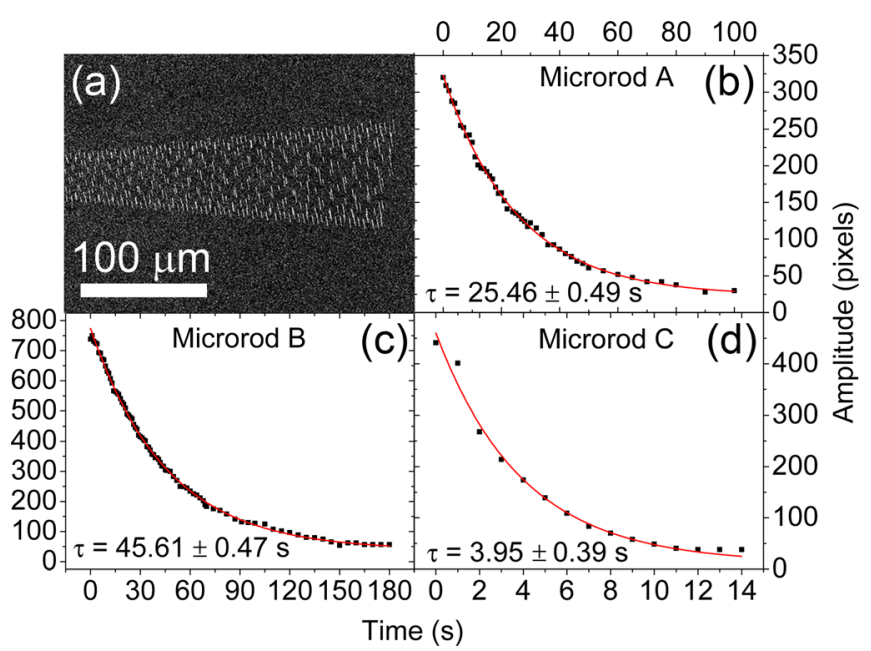

FIG. 3. (a) SEM image of an oscillating rod. (b)-(d) Experimentally measured decay curves (dots) and fitting to an exponential decay function (solid line). The decay constant is also shown for each curve. and are also listed in Table I. The Q-factors of these microrods were determined from the equation,

$$
Q=\pi \nu_{0} \tau
$$

where $\nu_{0}$ is the resonance frequency and $\tau$ is the oscillator decay constant.

The Q-factors, calculated from Equation (1), were found to be in the range of $10^{4}-10^{5}$ (see Table I), which are about two orders of magnitude higher than those previously measured in the microrods clamped with silver paint, and comparable to some of the best cantilever-like mechanical micro and nanoresonators made of diamond or $\mathrm{Si}$, which have $\mathrm{Q}$ factors in the range between $10^{4}$ and $10^{6} .^{14-16,36,37}$ These results evidence the importance of the intrinsic losses produced by radiation of energy through the clamp in $\mathrm{In}_{2} \mathrm{O}_{3}$ microrods, which are extensive to other material systems in a similar cantilever-configuration. Other intrinsic losses, such as the thermoelastic damping due to the heat flow produced by the material's periodic compression and expansion, surface losses due to surface roughness or material damping due to elastic energy dissipation, e.g., motion of lattice defects, ${ }^{16,18}$ can also limit the quality factor of the rods; however, the determination of their contribution to the total energy losses is not straightforward and requires further study.

In order to study the effect of extrinsic damping on the resonators behavior, which is mainly due to frictional process between the resonator and the surrounding atmosphere or due to the adsorption of molecules, measurements were performed at low-vacuum using the SEM environmental operation mode. In this mode, the working pressure is raised to values of the order of $10^{-1}$ Torr $(\sim 10 \mathrm{~Pa})$ using a $\mathrm{N}_{2}$ gas source. The low vacuum produces a fast damping of the rod's oscillation, thus hindering the measurement of their decay curves. Therefore, a different approach was used to determine the Q-factor value, taking advantage of its dependence with the damping coefficient $\gamma$

$$
Q=\frac{\nu_{0}}{2 \gamma} \text {. }
$$

The damping coefficient can be determined by fitting the amplitude vs. frequency curves to the equation of a 
one-dimensional harmonic resonator. ${ }^{30}$ Figure 4(a) shows the amplitude vs. frequency curves obtained for the microrod $\mathrm{A}$ at three different pressures between 0.08 and 0.15 Torr. At pressures above 0.15 Torr the SEM images were very unstable, which prevents the proper measurement of the resonance amplitude. It can be observed that the resonance frequency is shifted towards lower values with respect to the value of $1457.9 \mathrm{~Hz}$, measured in high-vacuum conditions. This is probably a consequence of the increased adsorption of molecules in the surface of the rods at higher pressures, since, as demonstrated below, the system is still in the molecular regime, and therefore the effect of the friction over the resonant frequency should be, in principle, negligible. ${ }^{38}$ The obtained values of the Q-factor are in the range of $10^{3}$, which represents a decrease of two orders of magnitude with respect to the value obtained at high-vacuum conditions, and shows the importance of the extrinsic damping mechanisms even at relatively low pressures. The inverse of the calculated quality factor has been plotted versus the gas pressure in Figure 4(b). The linear dependence observed in the graph corresponds to the molecular regime, in which the mean free path of the molecules is larger than the rods' oscillation amplitude. ${ }^{36}$ In this regime the extrinsic damping can be determined as ${ }^{36,38}$

$$
\frac{1}{Q_{e}}=\frac{p A}{2 \pi \nu_{0} m_{e f f} \nu},
$$

where $p$ is the gas pressure, $A$ is the lateral surface area, $m_{\text {eff }}$ is the effective mass of the resonator, and $v$ is the thermal velocity of the gas molecules, which is given by $v=\sqrt{k_{B} T / m}$, where $k_{B}$ is the Boltzmann constant, $T(=300 \mathrm{~K})$ is the temperature, and $m$ is the mass of the gas molecules, in our case $\mathrm{N}_{2}$. By fitting the experimental data in Eq. (3), the effective mass of microrod A was calculated to be $m_{\text {eff }}=5.41$ $\times 10^{-11} \mathrm{~kg}$. For the calculation of $A$, the rod cross section $S$ was assumed to vary linearly along the longitudinal axis $x$ (taking the base of the rod as $x=0)$ as $S=\left[\left(S_{T}-S_{B}\right) / l\right]$ $x+S_{B}$, where $S_{B}$ and $S_{T}$ are the cross sectional area at the bottom and the tip, respectively, and $l$ is the length of the rod.

The Q-factor of a mechanical oscillator strongly depends on its size, rapidly decreasing as the size decreases. ${ }^{16,33,36}$ Therefore, in order to effectively compare the sensitivity of two different resonators it is more practical to use the minimum detectable force than the $\mathrm{Q}$ factor. It represents the minimum force per bandwidth unit needed to produce a response in the resonator distinguishable from the thermal noise, and it is an important figure of merit commonly used to characterize the performance of mechanical micro- and nanoresonator sensors. ${ }^{14}$ It is given by

$$
F_{\min }=\sqrt{\frac{2 k_{r o d} k_{B} T}{\pi \nu_{0} Q}},\left[\mathrm{~N} \mathrm{~Hz}^{-1 / 2}\right],
$$

where $k_{\text {rod }}$ is the spring constant of the rod, $k_{B}$ is the Boltzmann constant, and $T$ is the temperature. The spring constant can be calculated from the resonance frequency and the effective mass by $k_{\text {rod }}=\left(2 \pi \nu_{0}\right)^{2} m_{\text {eff. }}$ In the case of microrods $\mathrm{B}$ and $\mathrm{C}$, it was not possible to determine the effective mass by the same procedure used for the microrod A due to the excessive damping of the rods during the environmental operation mode. Therefore, it was estimated by calculating the total mass of the rods from the indium oxide density and by using the same approximation for the cross section employed before. Thus,

$$
m_{e f f} \approx \frac{\left(S_{B}+S_{T}\right)}{2} l \rho,
$$

where $\rho=7179 \mathrm{~kg} / \mathrm{m}^{3}$ is the indium oxide density. The effective mass obtained for the microrod A using Eq. (3) $\left(m_{\text {eff }}=5.41 \times 10^{-11} \mathrm{~kg}\right)$ is of the same order of magnitude (less than a factor of two) to that obtained with Eq. (5) $\left(m_{\text {eff }}=3.01 \times 10^{-11} \mathrm{~kg}\right)$, meaning that this equation can be used to make a reasonable estimation of the effective mass of the microrods B and C. Calculated values of both the spring constant and the minimum detectable force are shown in Table I. The obtained values of the $F_{\min }$ are of the order of $10^{-16} \mathrm{~N} \mathrm{~Hz}^{-1 / 2}$, and are comparable to some of the best reported values for cantilever resonators at room temperature, ${ }^{3,4,14}$ which constitutes a remarkable result. One possible explanation for the low minimum detectable force is the lower Young's modulus of indium oxide $(E \sim 145 \mathrm{GPa})^{30}$ compared to diamond $(\sim 1200 \mathrm{GPa})^{39}$ or other materials such as $\mathrm{SiC}(E \sim 420 \mathrm{GPa})^{40}$ or $\mathrm{Si}_{3} \mathrm{~N}_{4}(\sim 280 \mathrm{GPa}),{ }^{41}$ which combined with the high $Q$, leads to a high sensitivity. Since the stiffness of a rectangular cross section rod increases with the third power of its thickness, it should be possible to further reduce the spring constant of the rods by decreasing their thickness, thus reducing their $F_{\min }$ even more. Our microrods have thicknesses which vary between several hundreds of nanometers to few tens of microns, while reported highly sensitive cantilever-like nanoresonators have thicknesses of
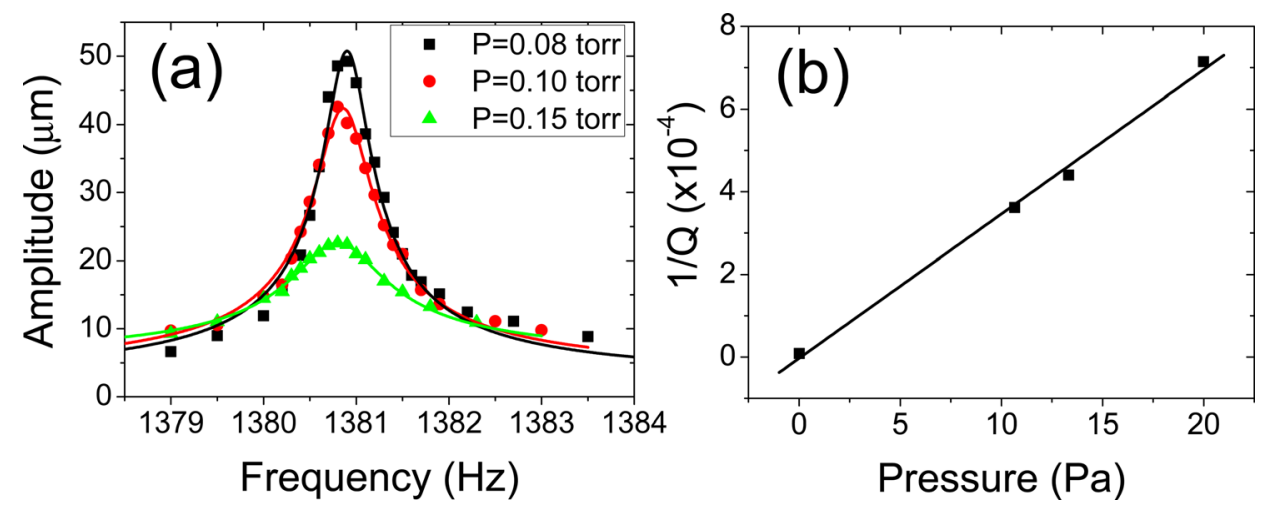

FIG. 4. (a) Resonance curves of micro$\operatorname{rod} \mathrm{A}$ measured at different pressures (dots) and fitting to the equation of a one-dimensional harmonic resonator equation (solid lines). (b) Corresponding plot of the inverse of the calculated Q-factor vs the measured pressure. 
the order of few hundreds of nanometers, meaning that there is still room for improvement. Besides, it has been demonstrated that not only the nature of the clamping but also its geometry greatly determines the amount of energy that is lost through the clamping. ${ }^{42,43}$ Therefore, lower values of $F_{\text {min }}$ should be achievable through the optimization of the size of the rods (by reducing their thickness) and their clamping geometry (by reducing its curvature radius or increasing the lateral size of the supporting medium).

To conclude, it has been shown that naturally clamped indium oxide microrods constitute a promising alternative for the fabrication of high $\mathrm{Q}$, high sensitivity mechanical microresonators for real applications such as force sensors for the detection of very weak forces as those required in several operating modes of atomic force microscopy or for extremely low mass detection, both of which can or need to operate in the pressure range studied in this work. An improvement of the Q-factor of two orders of magnitude compared to similar rods clamped with silver paint has been observed, obtaining Q-factors of the order of $10^{5}$. The estimated minimum detectable force is of the order of $10^{-16} \mathrm{~N}$ $\mathrm{Hz}^{-1 / 2}$, which is comparable to state-of-the-art cantileverlike mechanical micro and nanoresonators. It is expected that both the Q-factor and the minimum detectable force can be further improved by a proper optimization of the clamping geometry and the size of the rods.

This work has been supported by MINECO (Project Nos. MAT 2012-31959 and CSD 2009-00013). J.B. acknowledges the financial support from Universidad Complutense de Madrid.

${ }^{1}$ K. Eom, H. S. Park, D. S. Yoon, and T. Kwon, Phys. Rep. 503, 115 (2011).

${ }^{2}$ B. Ilic, D. Czaplewski, H. G. Craighead, P. Neuzil, C. Campagnolo, and C. Batt, Appl. Phys. Lett. 77, 450 (2000).

${ }^{3}$ M. Li, H. X. Tang, and M. L. Roukes, Nat. Nanotechnol. 2, 114 (2007).

${ }^{4}$ J. Moser, J. Güttinger, A. Eichler, M. J. Esplandiu, D. E. Liu, M. I. Dykman, and A. Bachtold, Nat. Nanotechnol. 8, 493 (2013).

${ }^{5}$ K. Jensen, K. Kim, and A. Zettl, Nat. Nanotechnol. 3, 533 (2008).

${ }^{6}$ J.-W. Han, J.-H. Ahn, M.-W. Kim, J. O. Lee, J.-B. Yoon, and Y.-K. Choi, Small 6, 1197 (2010).

${ }^{7}$ K. Jensen, J. Weldon, H. Garcia, and A. Zettl, Nano Lett. 7, 3508 (2007).

${ }^{8}$ M. Marzencki, J. Microelectromech. Syst. 18, 1444 (2009).

${ }^{9}$ T. J. Kippenberg, H. Rokhsari, T. Carmon, A. Scherer, and K. J. Vahala, Phys. Rev. Lett. 95, 033901 (2005).

${ }^{10}$ H. Rokhsari, T. Kippenberg, T. Carmon, and K. Vahala, IEEE J. Sel. Top. Quantum Electron. 12, 96 (2006).
${ }^{11}$ G. Anetsberger, R. Rivière, A. Schliesser, O. Arcizet, and T. J. Kippenberg, Nat. Photonics 2, 627 (2008).

${ }^{12}$ K.-K. Ni, R. Norte, D. J. Wilson, J. D. Hood, D. E. Chang, O. Painter, and H. J. Kimble, Phys. Rev. Lett. 108, 214302 (2012).

${ }^{13}$ H. Najar, M.-L. Chan, H.-A. Yang, L. Lin, D. G. Cahill, and D. A. Horsley, Appl. Phys. Lett. 104, 151903 (2014).

${ }^{14}$ Y. Tao, J. M. Boss, B. A. Moores, and C. L. Degen, Nat. Commun. 5, 3638 (2014).

${ }^{15}$ M. J. Burek, D. Ramos, P. Patel, I. W. Frank, and M. Lonar, Appl. Phys. Lett. 103, 131904 (2013).

${ }^{16}$ K. Y. Yasumura, T. D. Stowe, E. M. Chow, T. Pfafman, T. W. Kenny, B. C. Stipe, and D. Rugar, J. Microelectromech. Syst. 9, 117 (2000).

${ }^{17}$ T. Ono and M. Esashi, Meas. Sci. Technol. 15, 1977 (2004).

${ }^{18}$ S. Schmid and C. Hierold, J. Appl. Phys. 104, 093516 (2008).

${ }^{19}$ J. Bartolomé, A. Cremades, and J. Piqueras, J. Mater. Chem. C 1, 6790 (2013).

${ }^{20}$ C. Li, D. Zhang, S. Han, X. Liu, T. Tang, and C. Zhou, Adv. Mater. 15, 143 (2003).

${ }^{21}$ C.-J. Chen, M.-Y. Chern, C.-T. Wu, and C.-H. Chen, Mater. Res. Bull. 45, $230(2010)$

${ }^{22}$ Z. W. Pan, Z. R. Dai, and Z. L. Wang, Science 291, 1947 (2001).

${ }^{23}$ J. Jeong, J. Lee, C. Lee, S. An, and G.-C. Yi, Chem. Phys. Lett. 384, 246 (2004).

${ }^{24}$ B.-K. Lee, Y.-H. Song, and J.-B. Yoon, in Proceedings of IEEE 22nd International Conference on Indium Tin Oxide (ITO) Transparent MEMS Switches: Micro Electro Mechanical Systems, Sorrento, Italy, 25-29 January 2009, pp. 148-151.

${ }^{25}$ I. Hamberg and C. G. Granqvist, J. Appl. Phys. 60, R123 (1986).

${ }^{26}$ K. H. L. Zhang, A. Regoutz, R. G. Palgrave, D. J. Payne, R. G. Egdell, A. Walsh, S. P. Collins, D. Wermeille, and R. A. Cowley, Phys. Rev. B. 84, 233301 (2011).

${ }^{27}$ A. Walsh, C. Richard, A. Catlow, A. A. Alexey, A. Sokol, and S. M. Woodley, Chem. Mater. 21, 4962 (2009).

${ }^{28}$ D. Liu, W. W. Lei, B. Zou, S. D. Yu, J. Hao, K. Wang, B. B. Liu, Q. L. Cui, and G. T. Zou, J. Appl. Phys. 104, 083506 (2008).

${ }^{29}$ F. Fuchs and F. Bechstedt, Phys. Rev. B. 77, 155107 (2008).

${ }^{30}$ J. Bartolomé, P. Hidalgo, D. Maestre, A. Cremades, and J. Piqueras, Appl. Phys. Lett. 104, 161909 (2014).

${ }^{31}$ X. D. Bai, P. X. Gao, Z. L. Wang, and E. G. Wang, Appl. Phys. Lett. 82, 4806 (2003)

${ }^{32}$ C. Q. Chen, Y. Shi, Y. S. Zhang, J. Zhu, and Y. J. Yan, Phys. Rev. Lett. 96, 075505 (2006).

${ }^{33}$ S. Perisanu, V. Gouttenoire, P. Vincent, A. Ayari, M. Choueib, M. Bechelany, D. Cornu, and S. T. Purcell, Phys. Rev. B 77, 165434 (2008).

${ }^{34}$ Y. Hao, G. Meng, C. Ye, and L. Zhang, Cryst. Growth Des. 5, 1617 (2005).

${ }^{35}$ J. Y. Lao, J. G. Wen, and Z. F. Ren, Nano Lett. 2, 1287 (2002).

${ }^{36}$ K. L. Ekinci and M. L. Roukes, Rev. Sci. Instrum. 76, 061101 (2005).

${ }^{37}$ P. Ovartchaiyapong, L. M. A. Pascal, B. A. Myers, P. Lauria, and A. C. Bleszynski Jayich, Appl. Phys. Lett. 101, 163505 (2012).

${ }^{38}$ F. R. Blom, S. Bouwstra, M. Elwenspoek, and J. H. J. Fluitman, J. Vac. Sci. Technol. B 10, 19 (1992).

${ }^{39}$ R. Vogelgesang, A. K. Ramdas, S. Rodriguez, M. Grimsditch, and T. Anthony, Phys. Rev. B 54, 3989 (1996).

${ }^{40}$ Z. Li and R. C. Brandt, Int. J. High Technol. Ceram. 4, 1 (1988).

${ }^{41}$ A. Khan, J. Philip, and P. Hess, J. Appl. Phys. 95, 1667 (2004).

${ }^{42}$ S. Guillon, D. Saya, L. Mazenq, S. Perisanu, P. Vincent, A. Lazarus, O. Thomas, and L. Nicu, Nanotechnology 22, 245501 (2011).

${ }^{43}$ D. M. Photiadis and J. A. Judge, Appl. Phys. Lett. 85, 482 (2004). 